\title{
Mathematical simulation of temperature influence on pressure drop at pump suction line
}

\author{
Tomáš Polášek ${ }^{1, ~ *}$, Adam Bureček ${ }^{1}$, and Lumir Hružík ${ }^{1}$ \\ ${ }^{1}$ VŠB-Technical University of Ostrava, Faculty of Mechanical Engineering, Department of Hydromechanics and Hydraulic Equipment, \\ 70833 Ostrava, Czech Republic
}

\begin{abstract}
The article is focused on mathematical simulation of the temperature influence on the pressure drop at the pump suction line. It is evaluated pressure drop depending on mineral oil flow rate. The courses of individual dependencies are determined by mathematical simulation using the ANSYS CFD computing software. The temperature affects significantly physical properties of the flowing mineral oil. In a paper is also described the experimental measurement of a mineral oil sample and determination of its physical properties depending on temperature.
\end{abstract}

\section{Introduction}

The operating temperature of the mineral oil used in hydraulic machines and equipment significantly affects oil physical properties. It can have an essential influence on a correct operation of the equipment. For this reason, it is necessary to pay an increased attention to this issue.

The oil viscosity decreases with the increasing oil temperature in hydraulic mechanisms. In these cases, the flow losses are increased, pressure losses are reduced and the lubricating ability is worse with the decreasing oil viscosity.

It can be concluded that the viscosity is a measure of a liquid internal friction. Between two adjacent layers of a liquid which are moving with different velocities, the shear stress $\tau$ arises. According to the Newton's law for laminar flow, the shear stress $\tau$ is linearly proportional to the velocity gradient in a perpendicular direction to the direction of the flowing liquid. The shear stress $\tau$ is formulated in the following equation:

$$
\tau=\eta \cdot \frac{d v}{d y}
$$

where $\eta$ is dynamic viscosity, $\mathrm{d} v / \mathrm{d} y$ is velocity gradient in a perpendicular direction to the direction of movement.

The temperature influence is less pronounced on the change in the mineral oil density in comparison to the change in the oil viscosity. The mineral oil density decreases with the increasing operating temperature.

The determination of these two mineral oil physical properties in dependence on temperature is necessary to correctly define the flowing liquid in the mathematical model. This makes it possible to verify the oil temperature $t$ influence on the pressure drop $\Delta p$ when the mineral oil flows through the suction line of the pump. Gear pumps are very often used in mobile hydraulics where they are operated in heavy conditions. On the suction side, the significant negative vacuum is generated due to running at maximum operating parameters. The pumps are therefore extensively stressed by cavitation (aeration) erosion, resulting in a shorter service life and serviceability of the pump, noise level and vibration are increased and performance parameters are also reduced $[1,2]$.

\section{Experimental measurements}

Mineral oil physical properties are determined for the sent sample obtained from Jihostroj Company. For this sample, the oil dynamic viscosity $\eta$ dependence and oil density $\rho$ dependence were determined for the temperature range $t=(10 \div 80)^{\circ} \mathrm{C}$.

\subsection{Determination of the oil dynamic viscosity}

The Brookfield DV-II+Pro rotary viscometer was used to determine the viscous curve of the mineral oil sample. The aforementioned rotary viscometer operates on the principle of controlled shear rate and consist of a rotating spindle (type SEARLE). The small sample adapter was used to measure the mineral oil viscous curve. The rotary viscometer RV control was realized via Rheocalc V2.5 software. In order to achieve a constant temperature of the mineral oil sample, the rotary viscometer is equipped with a heat exchanger $\mathrm{HE}$ where the thermostat is included, see Figure 1.

Figure 1 shows the complete measuring device for determining mineral oil physical properties.

The selected spindle rotates with the measured oil in the small sample adapter. The oil dynamic viscosity is determined by the resistance, which is generated by the rotating spindle in the measured oil. 


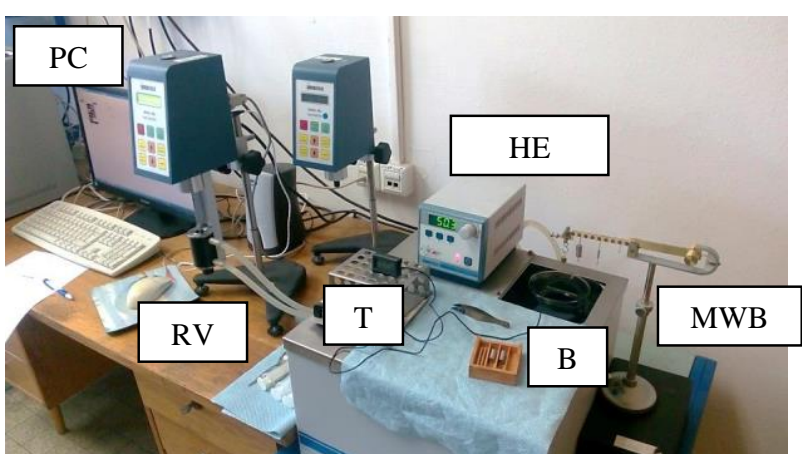

Fig. 1. Experimental measurements of the oil dynamic viscosity $\eta$ and the oil density $\rho$

The obtained viscous curve depending on the oil temperature $t$ is shown in Figure 2.

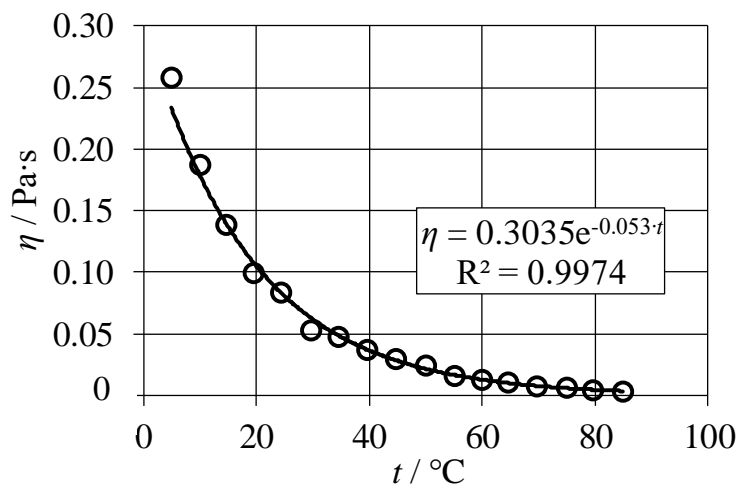

Fig. 2. Dependence of the oil dynamic viscosity $\eta$ on the temperature $t$

In Figure 2, it is possible to observe that the oil dynamic viscosity $\eta$ decreases significantly with the increasing temperature $t$ [3]. This mentioned viscosity decrease is more significant in the lower temperature range $t$.

\subsection{Determination of the oil density}

For determining the oil density $\rho$ dependence on the temperature $t$, the Mohr-Westphal balance MWB was used. The same heat exchanger $\mathrm{HE}$ as for the oil dynamic viscosity measurement was used to change the oil temperature in the beaker $\mathrm{B}$. The oil temperature $t$ was measured by the thermometer $\mathrm{T}$ in the beaker $\mathrm{B}$, see Figure 1.

Figure 3 shows the measured dependence of the oil density $\rho$ on the temperature $t$.

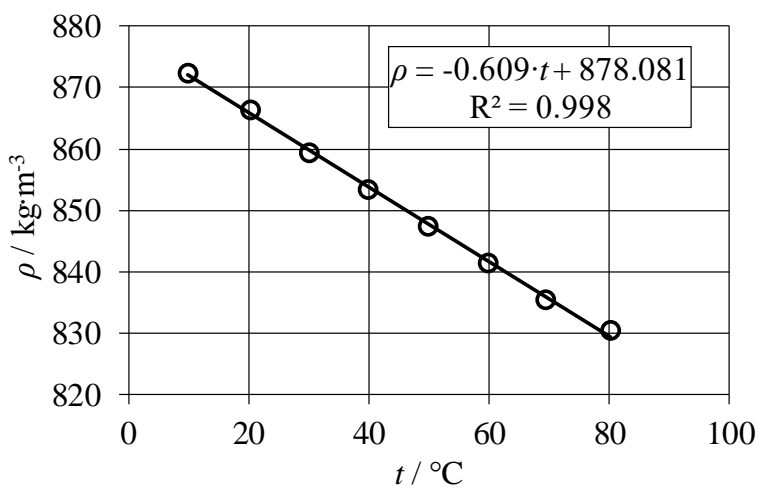

Fig. 3. Dependence of the oil density $\rho$ on the temperature $t$
In Figure 3, it can be seen that the oil density $\rho$ decreases with the increasing temperature $t$.

From these obtained dependencies of the oil physical properties on the temperature $t$, it is possible to define the flowing mineral oil for the wide range of temperatures $t$ using ANSYS CFX software.

Subsequently, the change in the pressure drop $\Delta p$ was monitored by changing the oil temperature $t$ at the suction line of the gear pump.

\section{Definition of the mathematical model and boundary conditions}

The geometry of the investigated external gear pump type GHD0-22.5R-S03D13-SU07U05- N was obtained from Jihostroj Company. The geometry preparation of the investigated flow domain was performed. Further, the computational mesh was created and boundary conditions were set up.

\subsection{Inverse geometry and computational mesh}

The DesignModeler environment was used to modify the geometry of the pump. All components that were irrelevant to the suction line flow investigation were removed from the model. The inverse geometry of the pump suction line was also created. The suction line parameters are the following:

- suction line length from the pump inlet $L=100 \mathrm{~mm}$,

- suction line inner diameter $d=26.8 \mathrm{~mm}$,

- depth of the fitting is included $h=14.5 \mathrm{~mm}$.

Figure 4 shows the inverse geometry of the suction line with the entire gear pump assembly.

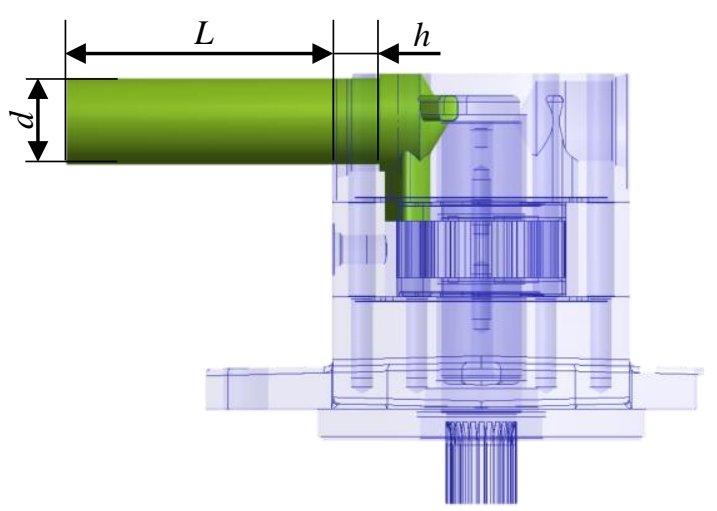

Fig. 4. The investigated flow domain

Then the computational mesh was created on the modified geometry. The mesh contained about 1000000 elements which that made up of tetrahedron elements.

The generated computational mesh was checked for an element deformation before another mathematical model setting and the numerical calculation running. For the evaluation, the skewness criterion was used. This criterion judges how close an element shape approaches an ideal geometric shape. The computational mesh, including boundary conditions of the investigated flow domain is shown in Figure 5. 


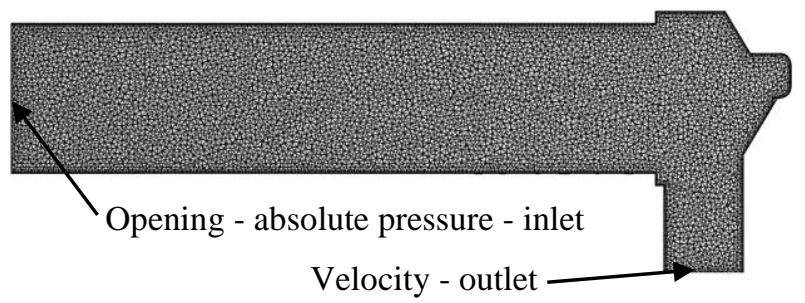

Fig. 5. The computational mesh and boundary conditions of the investigated flow domain

\subsection{Mathematical model and boundary conditions setting}

The numerical simulations were performed for flow velocities $v$, corresponding to the pump revolutions $n_{p}$, the outlet area $S$ and the geometric pump volume $V_{p}=22.5 \mathrm{~cm}^{3}$. The theoretical flow rate $Q_{p}$ of the investigated gear pump deals with the following expression:

$$
Q_{p}=V_{p} \cdot n_{p}
$$

The flow velocity $v$ can be determined by the continuity equation according to the expression:

$$
v=\frac{Q_{p}}{S}
$$

where the flow area $S$ depends on the shape and dimensions of the domain.

These flow simulations were performed for the oil temperature range $t=(10 \div 80){ }^{\circ} \mathrm{C}$. The flow velocities $v$, corresponding to the pump revolutions $n_{p}$ and the flow area (SL suction line - circular cross section, and outlet duct - non-circular cross section) are shown in Table 1.

Tab. 1. Flow velocities $v$ (outlet duct) and flow velocities $v_{S L}$ (suction line) corresponding to the pump revolutions $n_{p}$ and the flow area

\begin{tabular}{|c|c|c|}
\hline$n_{p} / \mathrm{min}^{-1}$ & $v / \mathrm{m} \cdot \mathrm{s}^{-1}$ & $v_{S L} / \mathrm{m} \cdot \mathrm{s}^{-1}$ \\
\hline 500 & 0.773 & 0.332 \\
\hline 1500 & 2.318 & 0.997 \\
\hline 2000 & 3.091 & 1.330 \\
\hline 3000 & 4.636 & 1.994 \\
\hline
\end{tabular}

At the entrance to the investigated domain, the Opening boundary condition, which was defined by the absolute pressure $p_{a b s}=101325 \mathrm{~Pa}$, was situated. Then at the outlet from the domain, the Velocity - outlet boundary condition was situated. This boundary condition was defined by the flow velocities $v$, corresponding to outlet duct, see Table 1.

The flowing liquid was defined based on the measured data that were obtained from the experimental measurements of the mineral oil physical properties, see Chapter 2.

The flow regime in the investigated domain was expressed by the Reynolds number Re. The values of the Reynolds numbers were determined by means of the oil dynamic viscosity $\eta$, the oil density $\rho$, the inner diameter $d$ of the suction line and the flow velocity $v_{S L}$ at the suction line. The analytical determination of the Reynolds number $R e$ is expressed as follows:

$$
R e=\frac{v_{S L} \cdot d \cdot \rho}{\eta} .
$$

The results of the Reynolds number $R e$ analytical determination depending on the oil temperature $t$ and the individual revolutions of the pump $n_{p}$ are shown in Figure 6.

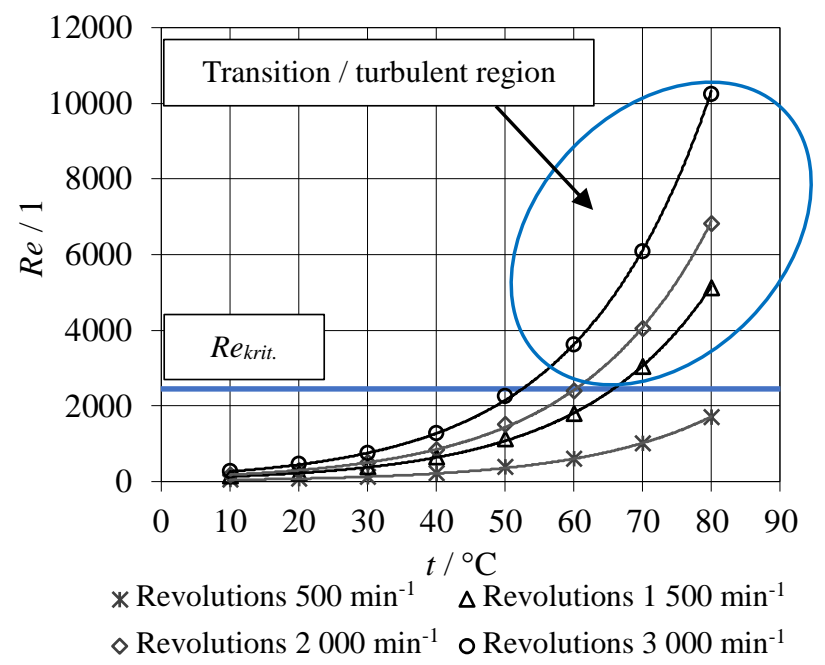

Fig. 6. Dependence of the Reynolds number $R e$ on the oil temperature $t$

The values of the Reynolds number Re correspond to laminar flow at most oil temperatures $t$. The Reynolds numbers $R e$ are higher than the critical value $R e_{k r i t}$ for the simulated revolutions $n_{p}=(1500,2000,3000) \mathrm{min}^{-1}$ and the oil temperatures $t=(60,70,80){ }^{\circ} \mathrm{C}$. Above the critical Reynolds number $R e_{k r i t}$, the flow is developing in a transition and a turbulent region. The laminar model was defined for the entire range of the oil temperatures $t$ and the flow velocities $v$.

\section{Evaluation of the numerical simulations}

Figure 7 shows velocity vectors in the symmetry plane of the investigated domain. The planes P1 and P2 are also shown in Figure between which the pressure drops $\Delta p$ of the suction line were determined.

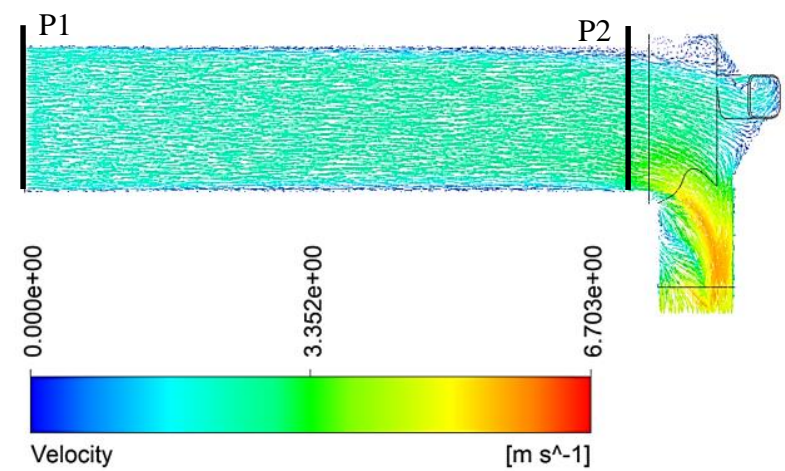

Fig. 7. Velocity vectors in the symmetry plane of the suction line for revolutions $n_{p}=3000 \mathrm{~min}^{-1}$, oil temperature $t=50{ }^{\circ} \mathrm{C}$, Laminar model 
The total pressure contours are shown in the symmetry plane and the suction line wall in Figure 8.

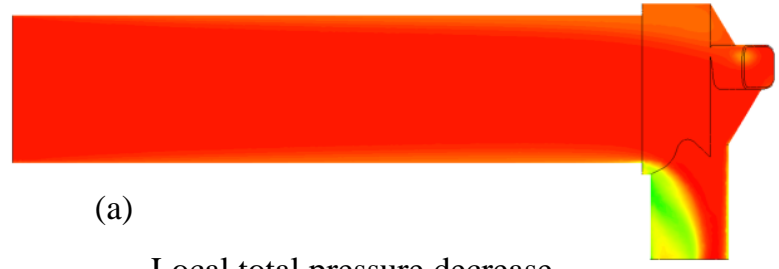

Local total pressure decrease on the suction line wall

(b)
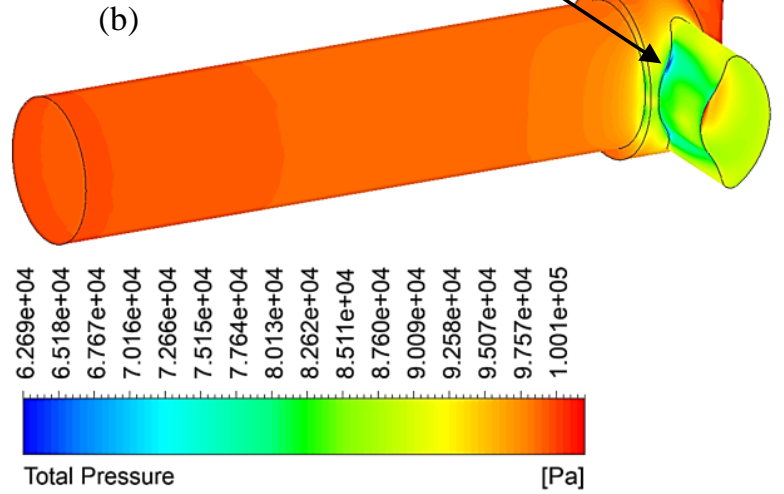

Fig. 8. Total pressure contours in the symmetry plane (a) and the suction line wall (b) for revolutions $n_{p}=3000 \mathrm{~min}^{-1}$, oil temperature $t=50^{\circ} \mathrm{C}$, Laminar model

The area of the largest total pressure decrease is also indicated on the suction line wall in Figure $8 \mathrm{~b}$. This area could then perform a significant role at the beginning of an air bubble formation $[4,5]$.

Figure 9 shows the pressure drops $\Delta p$ depending on the oil temperatures $t$ and the pump revolutions pump $n_{p}$ that were evaluated for the laminar model.

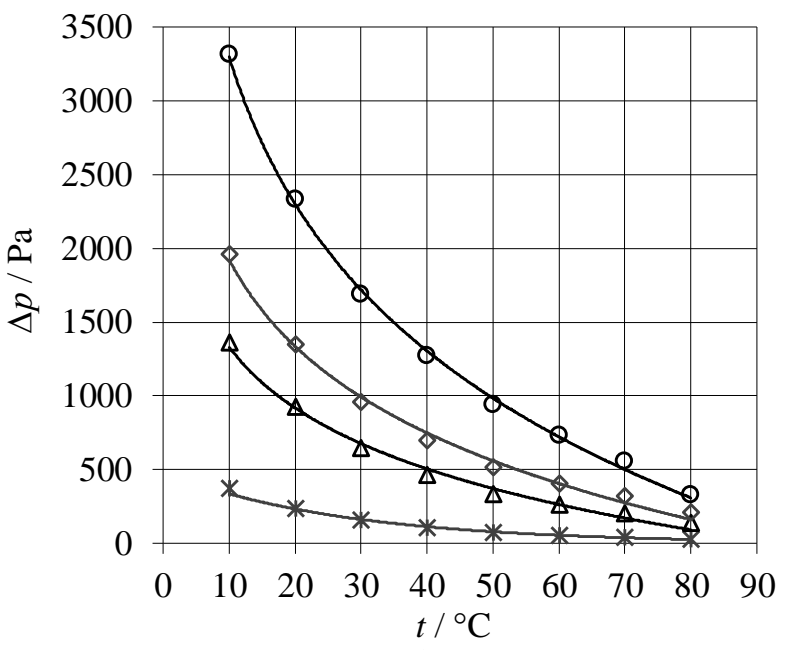

*Laminar model $500 \mathrm{~min}^{-1} \Delta$ Laminar model $1500 \mathrm{~min}^{-1}$

$\diamond$ Laminar model $2000 \mathrm{~min}^{-1}$ OLaminar model $3000 \mathrm{~min}^{-1}$

Fig. 9. Dependence of the pressure drop $\Delta p$ on the oil temperature $t$ and pump revolutions $n_{p}$ for the laminar model

In Figure 9, it is possible to observe that the pressure drop $\Delta p$ decreases with the increasing oil temperature $t$ at the suction line. It is also visible in Figure 9 that the pressure drop of the suction line $\Delta p$ increases with the increasing pump revolutions $n_{p}$ (the flow velocity $v$ ).
For higher oil temperatures $t=(60,70,80){ }^{\circ} \mathrm{C}$ and pump revolutions $n_{p}=(1500,2000,3000) \mathrm{min}^{-1}$, there wasn't a convergence of the laminar model.

For these cases, the mathematical simulations were performed for turbulent $k-\omega$, SST, $k-\varepsilon$ and RNG models. It was done by comparing the pressure drop $\Delta p$ depending on the oil temperature $t$ for a transition/turbulent flow regime [6].

Figure 10 shows numerical simulation results for selected turbulent models. The laminar model dependence $\Delta p=f(t)$ and aforementioned turbulent models dependencies $\Delta p=f(t)$ are compared for the pump revolutions $n_{p}=3000 \mathrm{~min}^{-1}$ and the oil temperatures $t=(60,70,80){ }^{\circ} \mathrm{C}$.

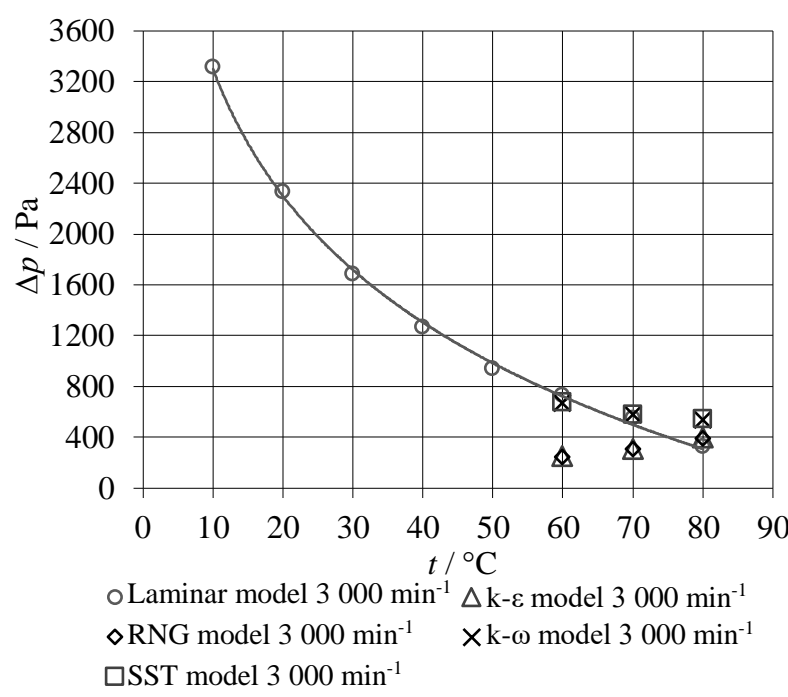

Fig. 10. Dependence of the pressure drop $\Delta p$ on the oil temperature $t$ and the pump revolutions $n_{p}=3000 \mathrm{~min}^{-1}$ for the laminar and turbulent models

The dependencies $\Delta p=f(t)$ determined by turbulent SST and $k-\omega$ models approximate with their trends to the dependence $\Delta p=f(t)$ determined by the laminar model. For the turbulent $k-\varepsilon$ and RNG models, the results differ significantly at oil temperatures $t=60$ a $70^{\circ} \mathrm{C}$. In Figure 10 , it is possible to observe that the pressure drops $\Delta p$ determined by the turbulent $k-\varepsilon$ and RNG models approximate to pressure drops $\Delta p$ determined by the turbulent SST and $k-\omega$ models with the increasing oil temperature $t$ (and therefore the increasing Reynolds number $R e$ ).

The use of the turbulent SST and $k-\omega$ models in ANSYS CFX software is suitable in a transition flow region.

\section{Conclusion}

The article deals with mathematical simulations of the oil temperature influence on the pressure drop at the suction line of the external gear pump. The mineral oil physical properties were experimentally determined. The mathematical flow model was created in the ANSYS CFX environment. The Reynolds numbers were determined by the analytical calculation depending on the oil temperature. The numerical solution result was decreasing dependence of the pressure drop on the oil 
temperature for the laminar model. In some cases, the Reynolds numbers reached higher values than the critical value. The simulations were performed for this area using the turbulent $k-\omega$, SST, $k-\varepsilon$, RNG models. The turbulent SST and $k-\omega$ models most closely approximated with their results to the dependence $\Delta p=f(t)$ which was determined by the laminar model.

This work was supported by the European Regional Development Fund in the Research Centre of Advanced Mechatronic Systems project, project number CZ.02.1.01/0.0/0.0/16_019/0000867 within the Operational Programme Research, Development and Education.

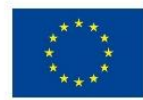
European Structural and Investment Funds Operational Programme Research, Development and Education

The work presented in this paper was supported by a grant SGS „Modeling and Experimental Verification of Dynamic Phenomena in Fluid and Vacuum Systems" SP2018/157.

\section{References}

1. R. Sikora, A. Bureček, L. Hružík, M. Vašina, EPJ Web of Conferences, EFM14, Experimental investigation of cavitation in pump inlet, 92, 02081, (2015)

2. M. Kozubková, M. Bojko, J. Jablonská, D. Homa, J. Tůma, EPJ Web of Conferences, EFM15, Experimental research of multiphase flow with cavitation in the nozzle, 114, 02058, (2016)

3. D. Knežević, V. Savić, FACTA UNIVERSITATIS, Mechanical Engineering, Mathematical modeling of changing of dynamic viscosity, as a function of temperature and pressure, of mineral oils for hydraulic systems, 4 (1), 27-34 p., (2006)

4. K. Fraňa, I. Nová, International Journal of Mechanics, A interface formation in two-phase flow problems, $\mathbf{1 2}$, 102-108p., (2018)

5. J. Jablonská, M. Bojko, EPJ Web of Conferences, EFM15, Mathematical and experimental modelling of flow of air-saturated water through a convergentdivergent nozzle, 114, 02050, (2016)

6. E. Toubiana, S. Russeil, D. Bougeard, N. François, Applied Thermal Engineering, Large Eddy simulation and Reynolds-averaged Navier-Stokes modeling of flow in staggered plate arrays: Comparison at various flow regimes, 89, 405-420 p., (2015) 